\title{
Nonlinear Nanofluid Flow over Heated Vertical Surface with Sinusoidal Wall Temperature Variations
}

\author{
S. S. Motsa, ${ }^{1}$ F. G. Awad, ${ }^{2}$ and M. Khumalo ${ }^{2}$ \\ ${ }^{1}$ School of Mathematical Sciences, University of KwaZulu-Natal, Private Bag X01, Scottsville, Pietermaritzburg 3209, South Africa \\ ${ }^{2}$ Department of Pure and Applied Mathematics, University of Johannesburg, P.O. Box 524, Auckland Park, \\ Johannesburg 2006, South Africa
}

Correspondence should be addressed to S. S. Motsa; sandilemotsa@gmail.com

Received 15 July 2014; Revised 15 August 2014; Accepted 15 August 2014; Published 14 October 2014

Academic Editor: Santanu Saha Ray

Copyright (c) 2014 S. S. Motsa et al. This is an open access article distributed under the Creative Commons Attribution License, which permits unrestricted use, distribution, and reproduction in any medium, provided the original work is properly cited.

\begin{abstract}
The nonlinear density temperature variations in two-dimensional nanofluid flow over heated vertical surface with a sinusoidal wall temperature are investigated. The model includes the effects of Brownian motion and thermophoresis. Using the boundary layer approximation, the two-dimensional momentum, heat, and mass transfer equations are transferred to nonlinear partial differential equations form and solved numerically using a new method called spectral local linearisation method. The effects of the governing parameters on the fluid properties and on the heat and nanomass transfer coefficients are determined and shown graphically.
\end{abstract}

\section{Introduction}

In recent years, nanofluids have attracted a considerable amount of interest due to their novel properties that make them potentially useful in a number of industrial applications including transportation, power generation, micromanufacturing, thermal therapy for cancer treatment, chemical and metallurgical sectors, heating, cooling, ventilation, and airconditioning. The term nanofluid is used to refer to a solidliquid mixture with a continuous phase in which nanoscale particles are dispersed in conventional base fluids. The nanofluid reference was first used by Choi [1]. The nanofluid can be readily configured either in a low state, where it conducts heat poorly, or in a high state, where the dissipation is more efficient [2]. The nanoparticles are made of various materials such as oxide ceramics, nitride ceramics, carbide ceramics, metals, semiconductors, and composite materials such as alloyed nanoparticles while the base fluids are usually water, ethylene glycol, toluene, and oil $[3,4]$. They are also important for the production of nanostructured materials for the engineering of complete fluids and for cleaning oil from surfaces due to their excellent wetting and spreading behaviour [4, 5]. Nanofluids can also be used as smart materials working as a heat valve to control the flow of heat.
A good review of the literature on nanofluids can be found in Buongiorno [6]. Kuznetsov and Nield [7] investigated the problem of natural convective boundary-layer flow of a nanofluid past a vertical plate. Their model considered the nanoparticle Brownian motion and thermophoresis with simple boundary conditions; namely, both the temperature and the nanoparticle fraction were constant along the wall. Using the Darcy model, Nield and Kuznetsov [8] studied the Cheng-Minkowycz problem of natural convection past a vertical plate in a porous medium saturated with a nanofluid. Khan and Pop [9] considered the effects of Brownian motion and thermophoresis in the laminar fluid flow from a stretching flat surface in a nanofluid. An experimental study of the problem was made for flow in vertical square enclosures of different sizes by Ho et al. [10]. Similar investigations have been conducted by, among others, Khan and Aziz [11], Rashad et al. [12], Aziz et al. [13], Rana et al. [14], Hajipour and Molaei Dehkordi [15], and Chanda and Rana [16].

The nonlinear temperature-density relationship in which the flow is driven by buoyancy may exert a strong influence on the flow and heat transfer characteristics. It is of practical importance in geothermal and engineering applications; for example, the residual warm water discharged from a geothermal power plant is usually disposed off 
from wells through subsurface reinjection. Using a parabolic temperature-density relationship, Korovkin and Andrievskii [17] investigated a fully developed free-convective flow above a horizontal linear heat source and on a flat impermeable vertical semi-infinite plate for three types of thermal boundary conditions, namely, an adiabatic surface, a constant temperature, and a constant heat flux on the surface. Vajravelu et al. [18] studied the problem of free convection flow at a vertical flat plate embedded in a saturated porous medium in the presence of heat sources (or sinks) with nonlinear density temperature variation. Fluid flow problems involving the nonlinear density temperature variation have been investigated by [19-22] among others. The recent study by Motsa et al. [23] investigated the problem of unsteady double diffusive convection flow over a stretching flat plate with nonlinear density temperature variation using a new version of the spectral homotopy analysis method.

The objective of this study is to consider the effects of the nonlinear density temperature variations as well as the Brownian motion and thermophoresis on nanofluid flow over heated vertical surface with a sinusoidal wall temperature. It is well known that power law surface temperature distributions give rise to self-similar boundary-layer flows (see $[24,25]$ ). To define the periodic array of heaters behind or within the wall, Rees [26] proposed another form of surface temperature variation with sinusoidal variations about the mean temperature which is held above the ambient temperature of the fluid. These types of boundary heating have been investigated by, among others, Pop and Ingham [27], Roy and Hossain [28], Saha et al. [29], Molla et al. [30], and Rana and Bhargava [31]. The momentum, heat, and mass transfer equations are reduced into nonlinear partial differential equations form. The high nonlinear systems governing the model are solved using a new method of solving systems of differential equations called the local linearisation method (LLM). The LLM is an innovative method that seeks to decouple systems of nonlinear differential equation and applies the Chebyshev spectral collocation in solving the resulting sequence of decoupled equations. The LLM was introduced by Motsa [32] for the solution of general nonlinear boundary-layer flow equations defined as systems of ordinary differential equations. The method has also been successfully used with domain transformation techniques in [33] to solve natural convection boundary-layer flow problem. In all the cited works, where the LLM has previously been applied, the governing equations were systems of ordinary differential equations. In this work we extend the application of the LLM to systems of nonlinear partial differential equations.

\section{Mathematical Formulation}

We consider nonlinear convection from a vertical flat surface embedded in a nanofluid of ambient temperature $\bar{T}_{\infty}$. We assume that the surface is kept at a steady temperature

$$
\bar{T}=\bar{T}_{\infty}+\left(\bar{T}_{w}-\bar{T}_{\infty}\right)\left(1+A \sin \left(\frac{\pi \bar{x}}{L}\right)\right)
$$

where $A$ is the relative amplitude of the surface temperature variations and $2 L$ is the wavelength of the variations, $\bar{T}_{w}>$ $\bar{T}_{\infty}$ for a heated surface and $\bar{T}_{w}<\bar{T}_{\infty}$ corresponding to a cooled surface. The temperature between the surface and the medium is assumed to be sufficiently large; hence the convection region is thick. In the buoyancy driven flow it is assumed that the density of the fluid $\rho$ depends nonlinearly on the temperature $\bar{T}$ of the fluid. In presence of Boussinesq's approximation the governing equations for the problem can be written in the form

$$
\begin{aligned}
& \frac{\partial \bar{u}}{\partial \bar{x}}+\frac{\partial \bar{v}}{\partial \bar{y}}=0, \\
& \rho_{f_{\infty}}\left[\bar{u} \frac{\partial \bar{u}}{\partial \bar{x}}+\bar{v} \frac{\partial \bar{u}}{\partial \bar{y}}\right]=\mu \frac{\partial^{2} \bar{u}}{\partial \bar{y}^{2}}+\left(1-\bar{\phi}_{\infty}\right) g \rho_{f_{\infty}} \\
& \times\left[\beta_{0}\left(\bar{T}-\bar{T}_{\infty}\right)+\beta_{1}\left(\bar{T}-\bar{T}_{\infty}\right)^{2}\right] \\
& -\left(\rho_{p}-\rho_{f_{\infty}}\right) g\left(\bar{\phi}-\bar{\phi}_{\infty}\right) \text {, } \\
& \bar{u} \frac{\partial \bar{T}}{\partial \bar{x}}+\bar{v} \frac{\partial \bar{T}}{\partial \bar{y}}=\alpha_{m} \frac{\partial \bar{T}}{\partial \bar{y}^{2}}+\tau\left[D_{B} \frac{\partial \bar{\phi}}{\partial \bar{y}} \frac{\partial \bar{T}}{\partial \bar{y}}+\frac{D_{T}}{\bar{T}_{\infty}}\left(\frac{\partial \bar{T}}{\partial \bar{y}}\right)^{2}\right], \\
& \bar{u} \frac{\partial \bar{\phi}}{\partial \bar{x}}+\bar{v} \frac{\partial \bar{\phi}}{\partial \bar{y}}=D_{B} \frac{\partial^{2} \bar{\phi}}{\partial \bar{y}^{2}}+\frac{D_{T}}{\bar{T}_{\infty}} \frac{\partial^{2} \bar{T}}{\partial \bar{y}^{2}},
\end{aligned}
$$

where $\bar{u}$ and $\bar{v}$ are the velocity components along the $x$ - and $y$-directions, respectively, $\bar{T}$ and $\bar{\phi}$ are the local fluid temperature and nanoparticle volume fraction, $\rho_{\infty}$ is the constant fluid density, $\bar{\phi}_{\infty}$ is the nanoparticle volume fraction, $\beta_{0}, \beta_{1}$ are thermal expansion coefficients, $\mu$ is the fluid viscosity, $\alpha_{m}$ is the effective thermal diffusivity, $D_{B}$ is the Brownian diffusion coefficient, $D_{T}$ is thermophoresis diffusion coefficient, $\rho_{f_{\infty}}$ is the fluid density, $\rho_{P}$ is particle mass density, $g$ is the acceleration due to the gravity, $\rho c_{P}$ is the effective heat capacity of the nanoparticle material, and $\tau$ is a parameter defined by $\rho c_{f} / \rho c_{P}$.

We introduce the following nondimensional variables:

$$
\begin{aligned}
& (x, y)=\frac{\left(\bar{x}, \bar{y} \mathrm{Gr}^{1 / 4}\right)}{L}, \quad(u, v)=\frac{\left(\bar{u}, \bar{v} \mathrm{Gr}^{1 / 4}\right)}{U_{c}} \\
& \frac{\bar{T}-\bar{T}_{\infty}}{\bar{T}_{w}-\bar{T}_{\infty}}=\theta, \quad \frac{\bar{\phi}-\bar{\phi}_{\infty}}{\bar{\phi}_{w}-\bar{\phi}_{\infty}}=\phi .
\end{aligned}
$$

The governing equations (2) can be presented as

$$
\begin{gathered}
\frac{\partial u}{\partial x}+\frac{\partial v}{\partial y}=0 \\
u \frac{\partial u}{\partial x}+v \frac{\partial u}{\partial y}=\frac{\partial^{2} u}{\partial y^{2}}+\theta+\sigma \theta^{2}-\operatorname{Nr} \phi
\end{gathered}
$$




$$
\begin{gathered}
u \frac{\partial \theta}{\partial x}+v \frac{\partial \theta}{\partial y}=\frac{1}{\operatorname{Pr}} \frac{\partial^{2} \theta}{\partial y^{2}}+\mathrm{Nb} \frac{\partial \phi}{\partial y} \frac{\partial \theta}{\partial y}+\mathrm{Nt}\left(\frac{\partial \theta}{\partial y}\right)^{2} \\
\operatorname{Le}\left(u \frac{\partial \phi}{\partial x}+v \frac{\partial \phi}{\partial y}\right)=\frac{\partial^{2} \phi}{\partial y^{2}}+\frac{\mathrm{Nt}}{\mathrm{Nb}} \frac{\partial^{2} \theta}{\partial y^{2}}
\end{gathered}
$$

subject to the boundary conditions

$$
\begin{gathered}
u=0, \quad v=0, \quad \theta=1+A \sin \pi x, \quad \phi=1 \\
\text { at } y=0, \\
u \longrightarrow 0, \quad \theta \longrightarrow 0, \quad \phi \longrightarrow 0 \quad \text { as } y \longrightarrow \infty .
\end{gathered}
$$

The various nondimensional parameters appearing in (4)-(7) are the nanofluid Grashof number Gr, the nonlinear temperature parameter $\sigma$, the buoyancy ratio $\mathrm{Nr}$, the Prandtl number $\mathrm{Pr}$, the Lewis number Le, the Brownian motion parameter $\mathrm{Nb}$, and the thermophoresis parameter Nt. These parameters are defined as

$$
\begin{gathered}
\mathrm{Gr}=\frac{\left(1-\phi_{\infty}\right) \beta_{0} g\left(\bar{T}_{w}-\bar{T}_{\infty}\right) L^{3}}{v^{2}}, \quad \sigma=\frac{\beta_{1}}{\beta_{0}}\left(\bar{T}_{w}-\bar{T}_{\infty}\right), \\
\mathrm{Nr}=\frac{\left(\rho_{p}-\rho_{f_{\infty}}\right)}{\left(1-\bar{\phi}_{\infty}\right) \beta_{0} \rho_{f_{\infty}}} \frac{\left(\bar{\phi}_{w}-\bar{\phi}_{\infty}\right)}{\left(\bar{T}_{w}-\bar{T}_{\infty}\right)}, \quad \operatorname{Pr}=\frac{\nu}{\alpha_{m}}, \\
\operatorname{Le}=\frac{\nu}{D_{B}}, \quad \mathrm{Nb}=\frac{\tau D_{B}\left(\bar{\phi}_{w}-\bar{\phi}_{\infty}\right)}{\nu}, \\
U_{c}=\frac{\nu}{L} \mathrm{Gr}^{1 / 2} .
\end{gathered}
$$

Introducing the stream function $\psi(x, y)$, where $u=\partial \psi / \partial y$ and $v=-\partial \psi / \partial x$, the two-dimensional equations (5)-(7) will take the form

$$
\begin{gathered}
\frac{\partial \psi}{\partial y} \frac{\partial^{2} \psi}{\partial x \partial y}-\frac{\partial \psi}{\partial x} \frac{\partial^{2} \psi}{\partial y^{2}}=\frac{\partial^{3} \psi}{\partial y^{3}}+\theta+\sigma \theta^{2}-\mathrm{Nr} \phi \\
\left(\frac{\partial \psi}{\partial y} \frac{\partial \theta}{\partial x}-\frac{\partial \psi}{\partial x} \frac{\partial \theta}{\partial y}\right)=\frac{1}{\operatorname{Pr}} \frac{\partial^{2} \theta}{\partial y^{2}}+\left[\mathrm{Nb} \frac{\partial \phi}{\partial y} \frac{\partial \theta}{\partial y}+\mathrm{Nt}\left(\frac{\partial \theta}{\partial y}\right)^{2}\right] \\
\operatorname{Le}\left(\frac{\partial \psi}{\partial y} \frac{\partial \phi}{\partial x}-\frac{\partial \psi}{\partial x} \frac{\partial \phi}{\partial y}\right)=\frac{\partial^{2} \phi}{\partial y^{2}}+\frac{\mathrm{Nt}}{\mathrm{Nb}} \frac{\partial^{2} \theta}{\partial y^{2}}
\end{gathered}
$$

The boundary conditions in (8) can be written in terms of the stream function as

$$
\begin{aligned}
& \frac{\partial \psi}{\partial x}=0, \quad \frac{\partial \psi}{\partial y}=0, \quad \theta=1 A \sin \pi x, \quad \phi=1 \\
& \text { at } y=0 \text {, } \\
& \frac{\partial \psi}{\partial y} \longrightarrow 0, \quad \theta \longrightarrow 0, \quad \phi \longrightarrow 0 \quad \text { as } y \longrightarrow \infty
\end{aligned}
$$

Equations (10) can further be simplified by introducing the following variables:

$$
\begin{gathered}
\psi=x^{3 / 4} f(x, \eta), \quad \theta=\theta(x, \eta), \quad \phi=\phi(x, \eta), \\
\eta=y x^{-1 / 4} .
\end{gathered}
$$

Using the similarity variables defined in (12), equations (10) along with the boundary conditions (11) reduce to the following two-dimensional boundary value problem:

$$
\begin{gathered}
\frac{\partial^{3} f}{\partial \eta^{3}}+\frac{3}{4} f \frac{\partial^{2} f}{\partial \eta^{2}}-\frac{1}{2}\left(\frac{\partial f}{\partial \eta}\right)^{2}+\theta+\sigma \theta^{2}-\mathrm{Nr} \phi \\
=x\left(\frac{\partial f}{\partial \eta} \frac{\partial^{2} f}{\partial x \partial \eta}-\frac{\partial^{2} f}{\partial \eta^{2}} \frac{\partial f}{\partial x}\right) \\
\frac{1}{\operatorname{Pr}} \frac{\partial^{2} \theta}{\partial \eta^{2}}+\frac{3}{4} f \frac{\partial \theta}{\partial \eta}+\left[\mathrm{Nb} \frac{\partial \phi}{\partial \eta} \frac{\partial \theta}{\partial \eta}+\mathrm{Nt}\left(\frac{\partial \theta}{\partial \eta}\right)^{2}\right] \\
=x\left(\frac{\partial f}{\partial \eta} \frac{\partial \theta}{\partial x}-\frac{\partial f}{\partial x} \frac{\partial \theta}{\partial \eta}\right) \\
\frac{1}{\operatorname{Le}} \frac{\partial^{2} \phi}{\partial \eta^{2}}+\frac{3}{4} f \frac{\partial \phi}{\partial \eta}+\frac{1}{\operatorname{Le}} \frac{\mathrm{Nt}}{\mathrm{Nb}} \frac{\partial^{2} \theta}{\partial \eta^{2}}=x\left(\frac{\partial f}{\partial \eta} \frac{\partial \phi}{\partial x}-\frac{\partial f}{\partial x} \frac{\partial \phi}{\partial \eta}\right)
\end{gathered}
$$

with the boundary conditions

$$
\begin{gathered}
f(x, 0)=0, \quad \frac{\partial f}{\partial \eta}(x, 0)=0, \\
\theta(x, 0)=1+A \sin \pi x, \quad \phi(x, 0)=1, \\
\frac{\partial f}{\partial \eta}(x, \infty)=0, \quad \theta(x, \infty)=0, \quad \phi(x, \infty)=0 .
\end{gathered}
$$

\section{Numerical Solution}

In this section we present the numerical method used to solve the governing nonlinear system of PDEs (13)-(16). The local linearisation method (LLM) approach is used to decouple the equations leading to a linear system which is subsequently solved using the Chebyshev spectral collocation method. The basic idea behind the LLM stems from the combination of the Gauss-Seidel method for decoupling equations and the Newton-Raphson based quasilinearisation. In this regard, linearisation in the momentum equation (13) is applied only in terms involving $f(\eta)$ and its derivatives. All other terms are assumed to be known from previous iterations. In the energy equation (14), linearisation is applied only to terms involving $\theta(\eta)$ and its derivatives. The terms involving $\phi(\eta)$ are assumed to be known from previous iteration while the updated solution for $\mathrm{f}(\eta)$ at the current iteration is used. Similarly, in the mass transfer equation (15), only terms in $\phi(\eta)$ are linearised while terms in $f(\eta)$ and $\theta(\eta)$ are assumed to be now known at the current iteration (denoted by $r+1$ ). In each case, the quasilinearisation is applied by assuming that the difference between the functions at the current and previous iterations is small. For example, in the case of $f(\eta)$, 
this difference is denoted by $f_{r+1}-f_{r}$. Thus applying the LLM on (13)-(16) gives

$$
\begin{aligned}
& f_{r+1}^{\prime \prime \prime}+a_{1, r} f_{r+1}^{\prime \prime}+a_{2, r} f_{r+1}^{\prime}+a_{3, r} f_{r+1}+a_{4, r} \\
&=a_{5, r} \frac{\partial f_{r+1}^{\prime}}{\partial x}+a_{6, r} \frac{\partial f_{r+1}}{\partial x}, \\
& \frac{1}{\operatorname{Pr}} \theta_{r+1}^{\prime \prime}+b_{1, r} \theta_{r+1}^{\prime}+b_{2, r}=b_{3, r} \frac{\partial \theta_{r+1}}{\partial x}, \\
& \frac{1}{\mathrm{Le}} \phi_{r+1}^{\prime \prime}+c_{1, r} \phi_{r+1}^{\prime}+c_{2, r}=b_{3, r} \frac{\partial \phi_{r+1}}{\partial x},
\end{aligned}
$$

where the primes denote partial derivatives with respect to $\eta$. The boundary conditions are given by

$$
\begin{gathered}
f_{r+1}(x, 0)=0, \quad f_{r+1}^{\prime}(x, 0)=0, \\
\theta_{r+1}(x, 0)=1+A \sin \pi x, \quad \phi_{r+1}(x, 0)=1, \\
f_{r+1}^{\prime}(x, \infty)=0, \quad \theta_{r+1}(x, \infty)=0, \quad \phi_{r+1}(x, \infty)=0 .
\end{gathered}
$$

The coefficients in (17)-(19) are defined as

$$
\begin{gathered}
a_{1, r}=\frac{3}{4} f_{r}+x \frac{\partial f_{r}}{\partial x}, \quad a_{2, r}=-f_{r}^{\prime}-x \frac{\partial f_{r}^{\prime}}{\partial x}, \quad a_{3, r}=\frac{3}{4} f_{r}^{\prime \prime}, \\
a_{4, r}=\theta_{r}+\sigma \theta_{r}^{2}-N_{r} \phi_{r}+\frac{1}{2}\left(f_{r}^{\prime}\right)^{2}-\frac{3}{4} f_{r} f_{r}^{\prime \prime} \\
+x\left(f_{r}^{\prime} \frac{\partial f_{r}^{\prime}}{\partial x}-f_{r}^{\prime \prime} \frac{\partial f_{r}}{\partial x}\right), \\
a_{5, r}=x f_{r}^{\prime}, \quad a_{6, r}=-x f_{r}^{\prime \prime}, \\
b_{1, r}=\frac{3}{4} f_{r}+N_{b} \phi_{r}^{\prime}+2 N_{t} \theta_{r}^{\prime}+x \frac{\partial f_{r}}{\partial x}, \\
b_{2, r}=-N_{t}\left(\theta_{r}^{\prime}\right)^{2}, \quad b_{3, r}=x f_{r}^{\prime}, \quad c_{1, r}=\frac{3}{4} f_{r}+x \frac{\partial f_{r}}{\partial x}, \\
c_{2, r}=\frac{1}{\operatorname{Le}} \frac{N_{t}}{N_{b}} \theta_{r}^{\prime}, \quad c_{3, r}=x f_{r}^{\prime} .
\end{gathered}
$$

To solve the linearised system of (17)-(18) we employ the Chebyshev spectral collocation method to discretize in the $\eta$ direction and use an implicit finite difference method in the $x$-direction. To this end, we define the grid points on $(\eta, x)$ as

$$
\begin{array}{r}
\eta_{j}=\cos \frac{\pi j}{N_{x}}, \quad x^{n}=n \Delta x, \\
j=0,1, \ldots, N_{x} ; n=0,1, \ldots, N_{t},
\end{array}
$$

where $N_{x}, N_{t}$ are the total number of grid points in the $\eta$ - and $x$-directions, respectively, and $\Delta x$ is the spacing in the $x$-direction. The finite difference scheme is applied with centering about a midpoint halfway between $x^{n+1}$ and $x^{n}$. This midpoint is defined as $x^{n+1 / 2}=\left(x^{n+1}+x^{n}\right) / 2$.
The derivatives with respect with $\eta$ are discretized in terms of the Chebyshev differentiation matrices. Applying the centering about $x^{n+1 / 2}$ to any function, say $f(\eta, x)$ and its associated derivative, we obtain

$$
\begin{gathered}
f\left(\eta_{j}, x^{n+1 / 2}\right)=f_{j}^{n+1 / 2}=\frac{f_{j}^{n+1}+f_{j}^{n}}{2}, \\
\left(\frac{\partial f}{\partial x}\right)^{n+1 / 2}=\frac{f_{j}^{n+1}-f_{j}^{n}}{\Delta x} .
\end{gathered}
$$

In applying the Chebyshev spectral collocation method, the continuous derivatives in the unknown functions are approximated by matrix-vector products of the so-called differentiation matrices at the collocation points. Before the spectral method is applied, the domain $\eta \in\left[0, \eta_{\infty}\right]$ is transformed to the domain $Y \in[-1,1]$ on which the spectral method can be implemented. Thus, the derivatives, say $f^{\prime}$, $f^{\prime \prime}$, at the collocation points $Y_{j}\left(j=0,1, \ldots, N_{x}\right)$, are given in matrix form in $[34,35]$ as a differentiation matrix $D$ that maps a vector of the function values $\mathbf{F}=\left[f\left(Y_{0}\right), \ldots, f\left(Y_{N_{x}}\right)\right]^{T}$ at the collocation points to a vector $\mathbf{F}^{\prime}$ defined as

$$
\mathbf{F}^{\prime}=\sum_{k=0}^{N_{x}} D_{j k} f\left(Y_{k}\right)=\mathbf{D F}, \quad j=0,1, \ldots, N_{x} .
$$

In general, a derivative of order $s$ for the function $f(\eta)$ can be transformed as

$$
f^{(s)}(\eta) \longrightarrow \mathbf{D}^{s} \mathbf{F}
$$

Thus, applying the spectral method in $\eta$ and finite difference method in $x$ gives

$$
\begin{aligned}
& A_{1} \mathbf{F}_{r+1}^{n+1}=B_{1} \mathbf{F}_{r+1}^{n}+K_{1}, \\
& A_{2} \Theta_{r+1}^{n+1}=B_{2} \Theta_{r+1}^{n}+K_{2}, \\
& A_{3} \boldsymbol{\Phi}_{r+1}^{n+1}=B_{3} \boldsymbol{\Phi}_{r+1}^{n}+K_{3},
\end{aligned}
$$

where

$$
\begin{aligned}
A_{1}= & \frac{1}{2}\left(\mathbf{D}^{3}+\mathbf{a}_{1, r}^{n+1 / 2} \mathbf{D}^{2}+\mathbf{a}_{2, r}^{n+1 / 2} \mathbf{D}+\mathbf{a}_{3, r}^{n+1 / 2}\right) \\
& -\frac{\mathbf{a}_{5, r}^{n+1 / 2} \mathbf{D}}{\Delta \xi}-\frac{\mathbf{a}_{6, r}^{n+1 / 2}}{\Delta \xi}, \\
B_{1}= & -\frac{1}{2}\left(\mathbf{D}^{3}+\mathbf{a}_{1, r}^{n+1 / 2} \mathbf{D}^{2}+\mathbf{a}_{2, r}^{n+1 / 2} \mathbf{D}+\mathbf{a}_{3, r}^{n+1 / 2}\right) \\
& -\frac{\mathbf{a}_{5, r}^{n+(1 / 2)} \mathbf{D}}{\Delta \xi}-\frac{\mathbf{a}_{6, r}^{n+1 / 2}}{\Delta \xi}, \\
A_{2}= & \frac{1}{2}\left(\frac{1}{\operatorname{Pr}} \mathbf{D}^{2}+\mathbf{b}_{1, r}^{n+1 / 2} \mathbf{D}\right)-\frac{\mathbf{b}_{3, r}^{n+1 / 2}}{\Delta \xi} \\
B_{2}= & -\frac{1}{2}\left(\frac{1}{\operatorname{Pr}} \mathbf{D}^{2}+\mathbf{b}_{1, r}^{n+1 / 2} \mathbf{D}\right)-\frac{\mathbf{b}_{3, r}^{n+1 / 2}}{\Delta \xi}
\end{aligned}
$$




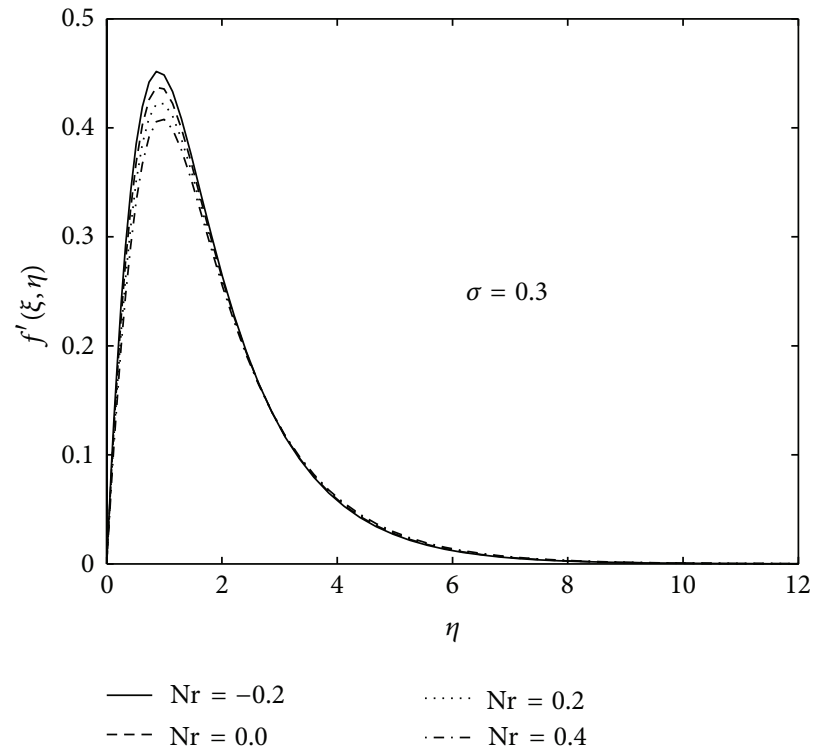

(a)

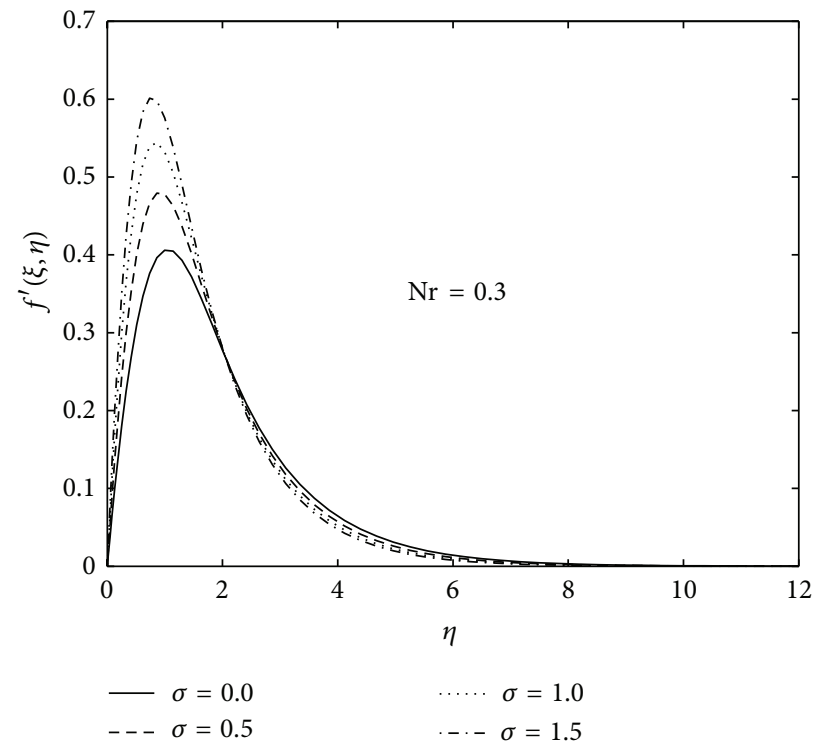

(b)

FIgURE 1: Effect of the buoyancy ratio $\mathrm{Nr}$ on $f(\eta)$ and $f^{\prime}(\eta)$ for $\xi=0.5, \mathrm{Le}=10, \mathrm{Pr}=7, \mathrm{Nt}=0.1, \mathrm{Nb}=0.5$, and $A=0.1$.

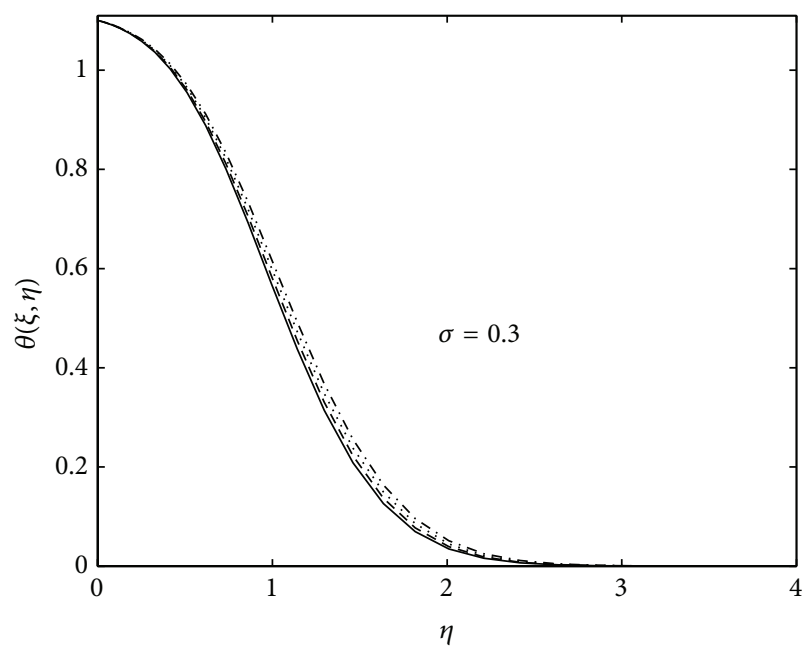

$$
\begin{aligned}
& -\mathrm{Nr}=-0.2 \\
& ---\mathrm{Nr}=0.0 \\
& \text { … } \mathrm{Nr}=0.2 \\
& \text {-.. } \mathrm{Nr}=0.4
\end{aligned}
$$

(a)

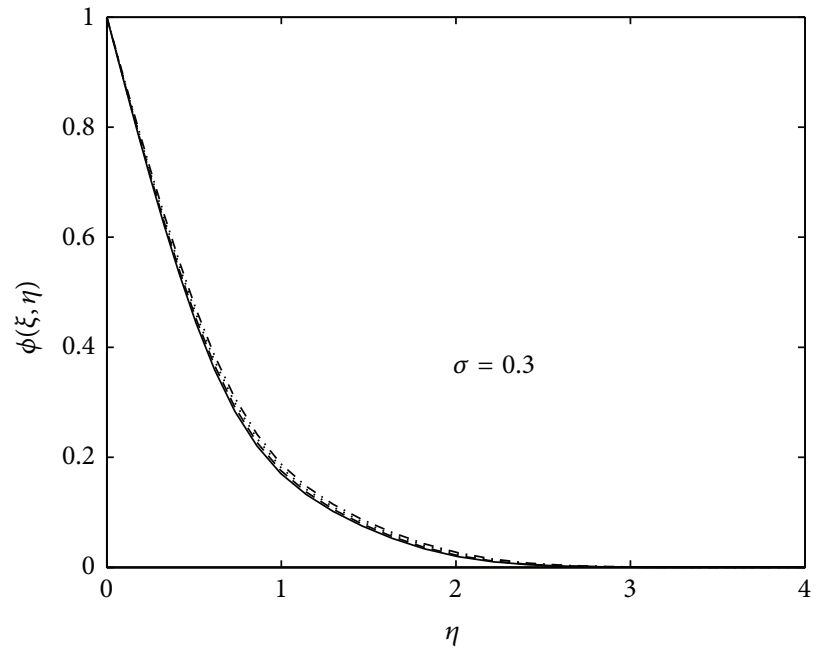

$$
\begin{aligned}
& -\mathrm{Nr}=-0.2 \\
& ---\mathrm{Nr}=0.0 \\
& \mathrm{Nr}=0.2
\end{aligned}
$$

(b)

Figure 2: Effect of the buoyancy ratio $\mathrm{Nr}$ on $\theta(\eta)$ and $\phi(\eta)$ for $\xi=0.5$, $\mathrm{Le}=10, \mathrm{Pr}=7, \mathrm{Nt}=0.1, \mathrm{Nb}=0.5$, and $A=0.1$.

$$
\begin{gathered}
A_{3}=\frac{1}{2}\left(\frac{1}{\mathrm{Le}} \mathbf{D}^{2}+\mathbf{c}_{1, r}^{n+1 / 2} \mathbf{D}\right)-\frac{\mathbf{c}_{3, r}^{n+1 / 2}}{\Delta \xi}, \\
B_{3}=-\frac{1}{2}\left(\frac{1}{\mathrm{Le}} \mathbf{D}^{2}+\mathbf{c}_{1, r}^{n+1 / 2} \mathbf{D}\right)-\frac{\mathbf{c}_{3, r}^{n+1 / 2}}{\Delta \xi}, \\
K_{1}=-\mathbf{a}_{4, r}^{n+1 / 2}, \quad K_{2}=-\mathbf{b}_{2, r}^{n+1 / 2}, \quad K_{3}=-\mathbf{c}_{2, r}^{n+1 / 2} .
\end{gathered}
$$

In the above equations $\boldsymbol{\Phi}, \boldsymbol{\Theta}$, and $\mathbf{F}$ correspond to the approximate values of $\phi(\eta, x), \theta(\eta, x)$, and $f(\eta, x)$ at the collocation points. The approximate solutions for $f, \theta$, and $\phi$ are obtained by solving (26). The convergence and stability of the iteration schemes are assessed by considering the norm of the difference in the values of the approximate functions between two successive iterations. Thus, for each iteration scheme, we define the following maximum error $E$ at the $(r+1)$ th iteration:

$$
E=\max \left(\left\|\mathbf{F}_{r+1}-\mathbf{F}_{r}\right\|_{\infty},\left\|\boldsymbol{\Theta}_{r+1}-\boldsymbol{\Theta}_{r}\right\|_{\infty},\left\|\boldsymbol{\Phi}_{r+1}-\boldsymbol{\Phi}_{r}\right\|_{\infty}\right) .
$$




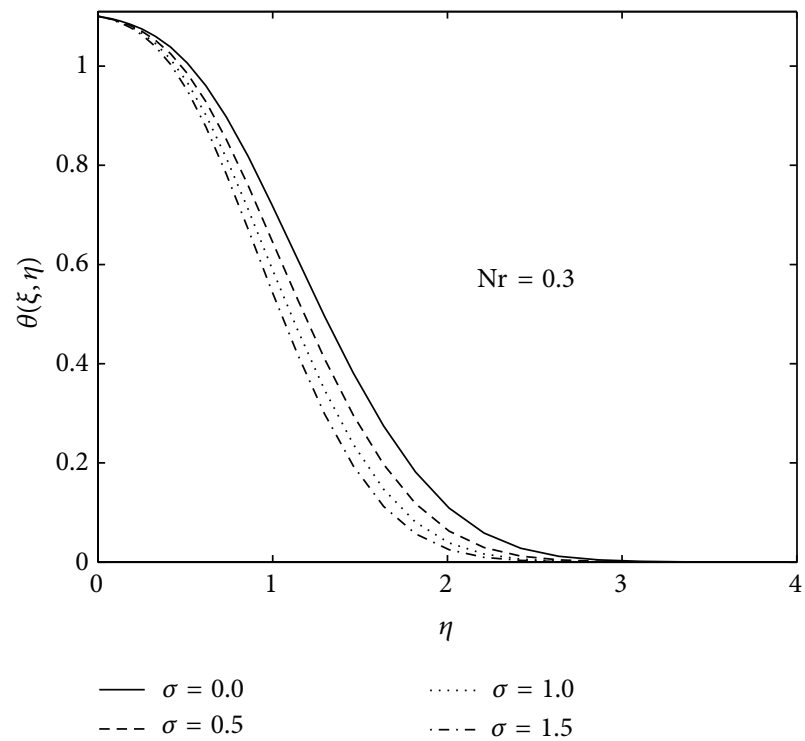

(a)

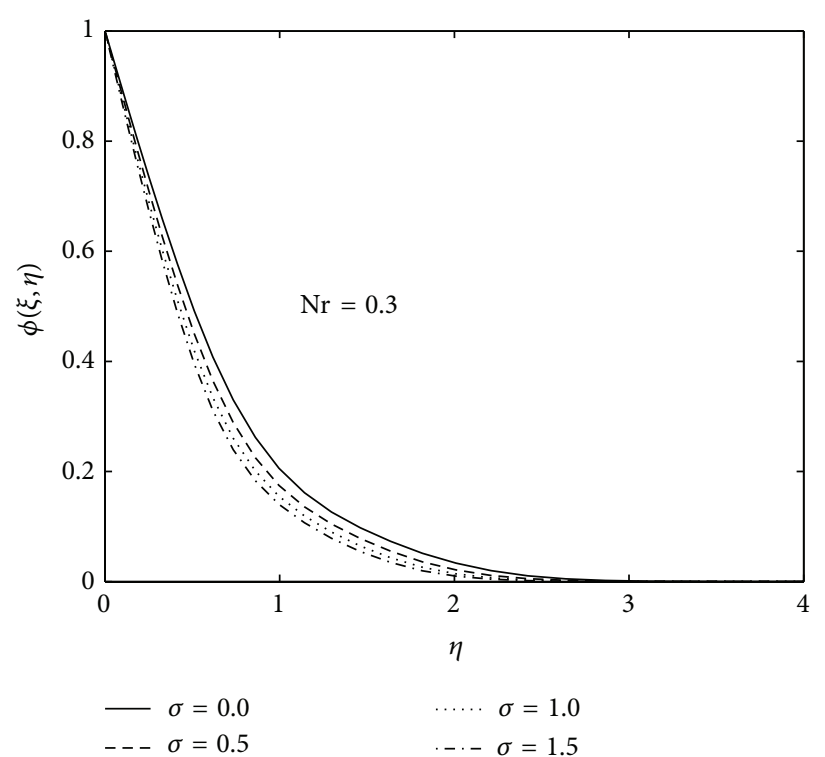

(b)

Figure 3: Effect of the $\sigma$ on $\theta(\eta)$ and $\phi(\eta)$ for $x=0.5, \mathrm{Le}=10, \mathrm{Pr}=7, A=0.1, \mathrm{Nr}=0.1, \mathrm{Nt}=0.1$, and $\mathrm{Nb}=0.5$.

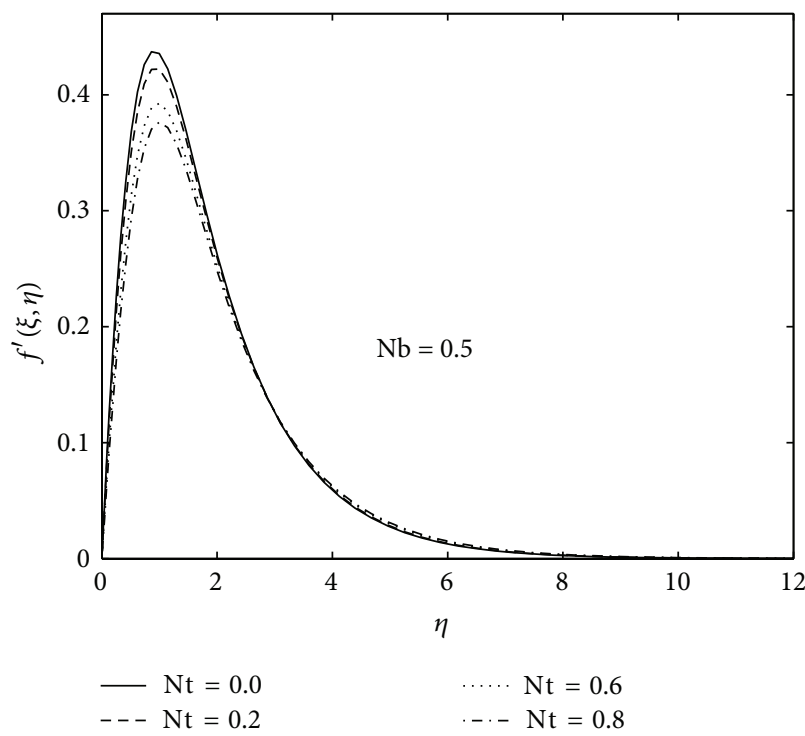

(a)

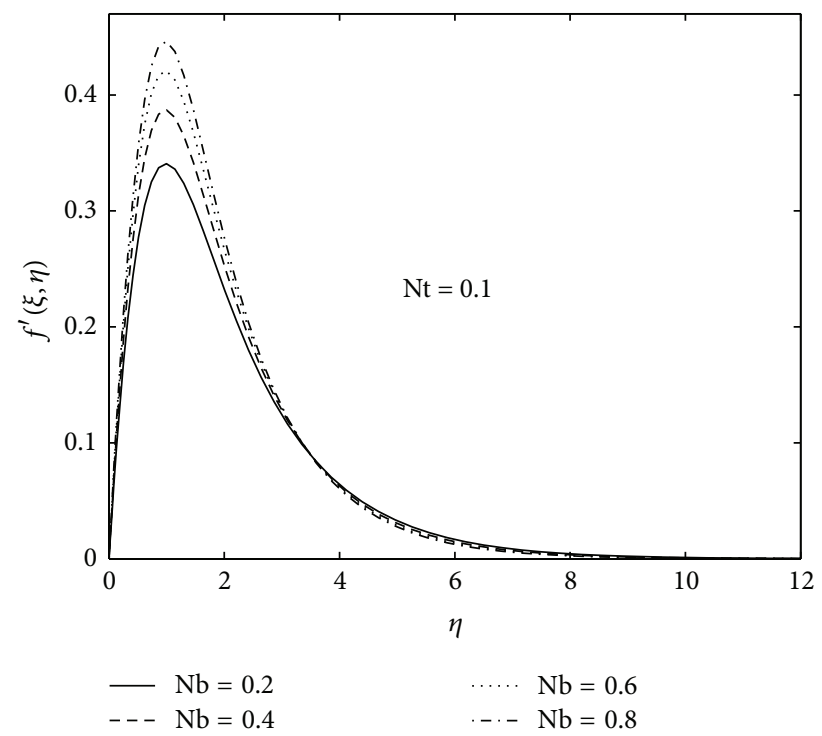

(b)

Figure 4: Effect of the Nt on $f^{\prime}(\eta)$ for $\xi=0.5, \mathrm{Le}=10, \mathrm{Pr}=7, \sigma=0.3, \mathrm{Nr}=0.1$, and $A=0.1$.

The unknowns $f, \theta$, and $\phi$ were iteratively calculated, for a given number of collocation points $N_{x}$, until the following criteria for convergence was satisfied at iteration $r$ :

$$
E \leq \epsilon
$$

where $\epsilon$ is the convergence tolerance level which was chosen to be $10^{-7}$ in this work. The results are given in the next section.

\section{Results and Discussions}

In this section we present solutions of (13)-(15) along with the boundary conditions (16) which were generated using the local linearisation method described in the previous section. In order to obtain clear insights into the physics of the problem of nanofluid flow over heated vertical surface with sinusoidal wall temperature variations in presence of the nonlinear temperature-density relationship, analysis has been carried out for different values of our governing parameters, 


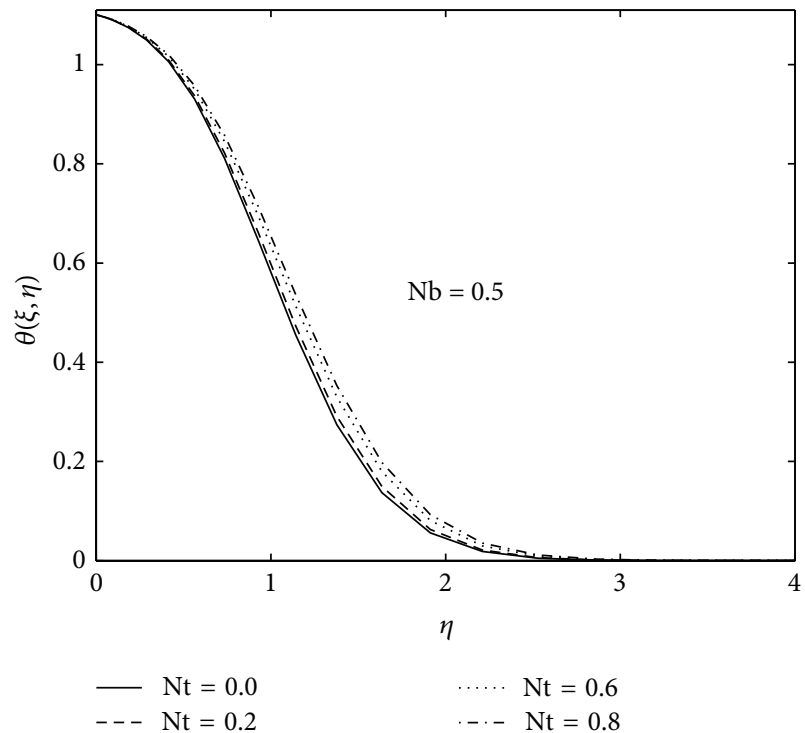

(a)

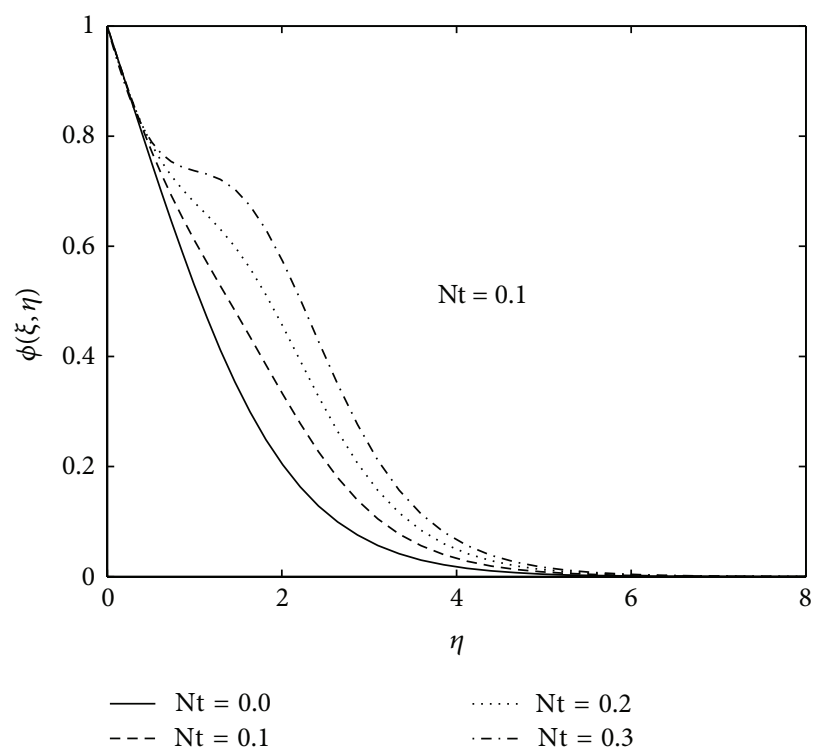

(b)

Figure 5: Effect of the Nt on $\theta(\eta)$ and $\phi(\eta)$ for $\xi=0.5, \mathrm{Le}=10, \operatorname{Pr}=7, \sigma=0.3, \mathrm{Nr}=0.1, \mathrm{Nb}=0.5$, and $A=0.1$.

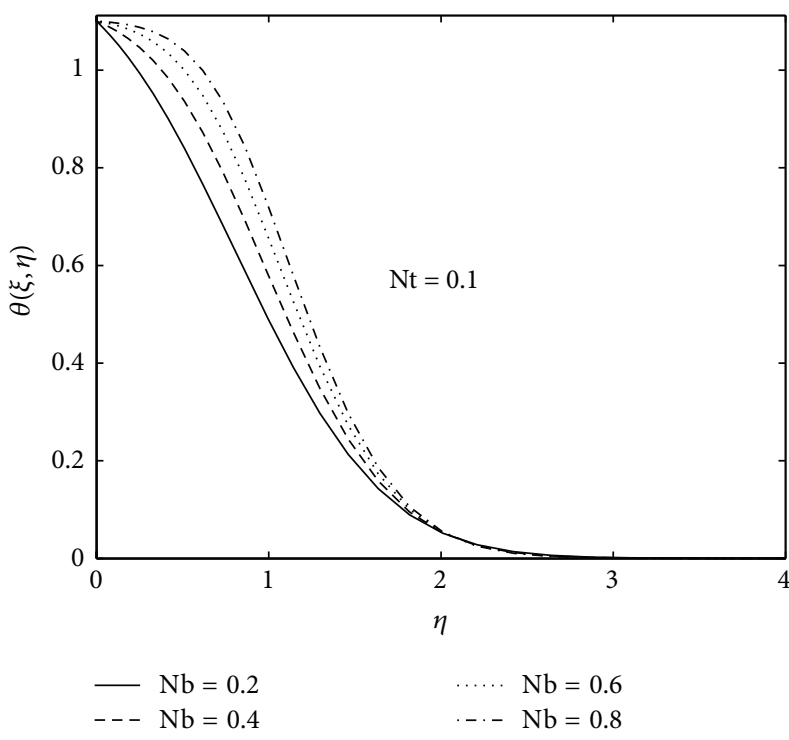

(a)

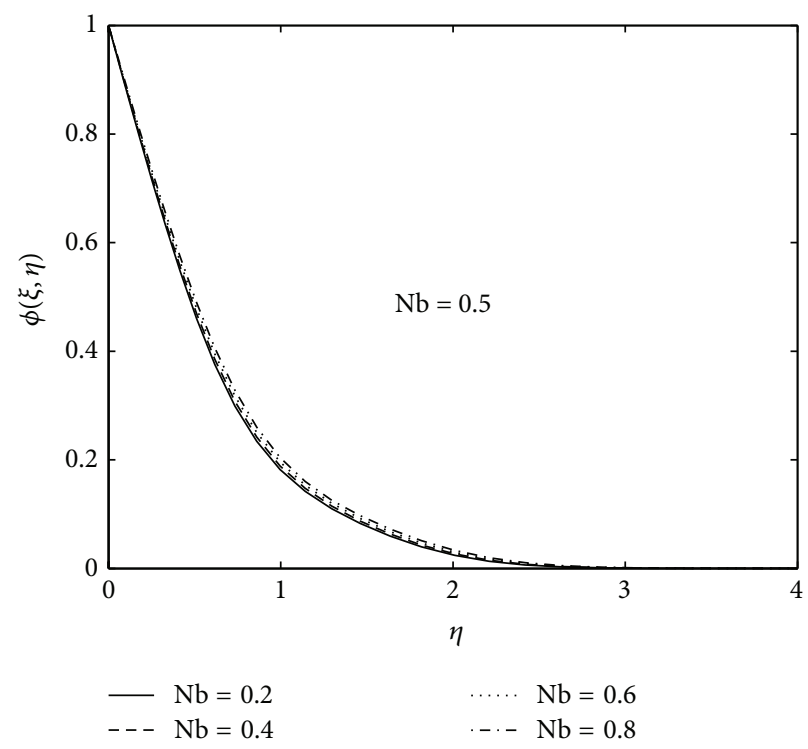

(b)

Figure 6: Effect of the $\mathrm{Nb}$ on $\theta(\eta)$ and $\phi(\eta)$ for $\xi=0.5, \mathrm{Le}=10, \mathrm{Pr}=7, \sigma=0.3, \mathrm{Nr}=0.1, \mathrm{Nt}=0.1$, and $A=0.1$.

namely, the nonlinear temperature parameter $\sigma$, the Buoyancy ratio $\mathrm{Nr}$, the Prandtl number $\mathrm{Pr}$, the Lewis number Le, the Brownian motion parameter $\mathrm{Nb}$, and the thermophoresis parameter $\mathrm{Nt}$ at some streamwise positions $x$. Table 1 gives a comparison between the present results and Rees [26] for the skin-friction and the heat transfer coefficient, respectively. Firstly we note a remarkable agreement between the two sets of results, and secondly as the Prandtl Pr increases, the skinfriction coefficient decreases and the rate of heat transfer increases with increasing Prandtl number.
Figure 1 shows the effect of increasing the buoyancy ratio and the nonlinear temperature parameter $\sigma$ on the velocity component $f^{\prime}(\eta)$. Increasing the buoyancy ratio parameter leads to increased thickness of the momentum boundary layer. We also observe that the velocity decelerates at the surface with an increase in the buoyancy ratio parameter. In addition, as $\sigma$ increases, the velocity tends to increase very closely to the surface near the edge of boundary layer in a narrow region. The nonlinear temperature parameter is expected to alter the momentum boundary layer; hence 


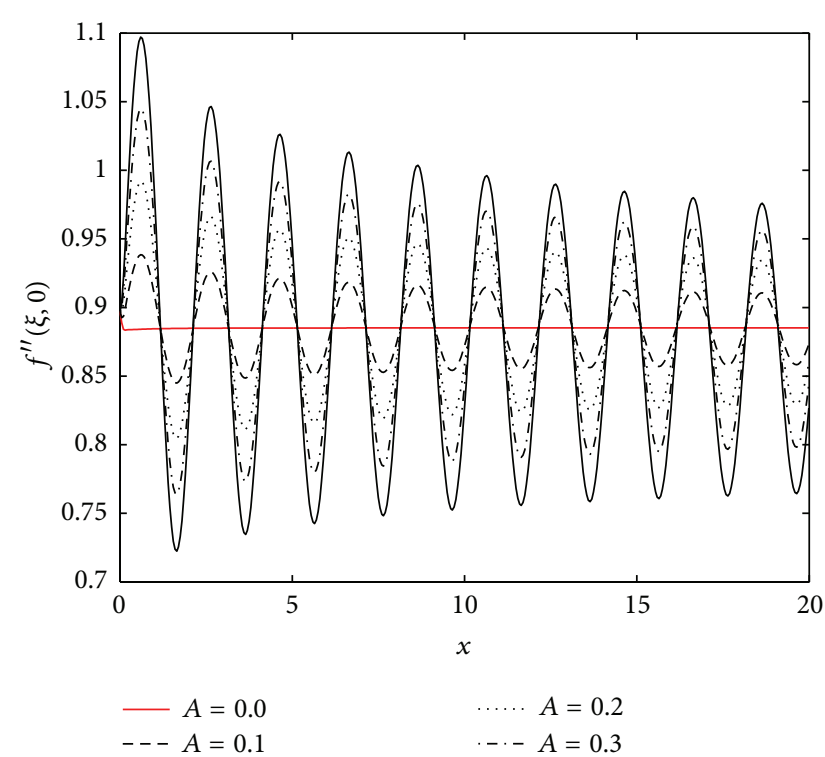

(a)

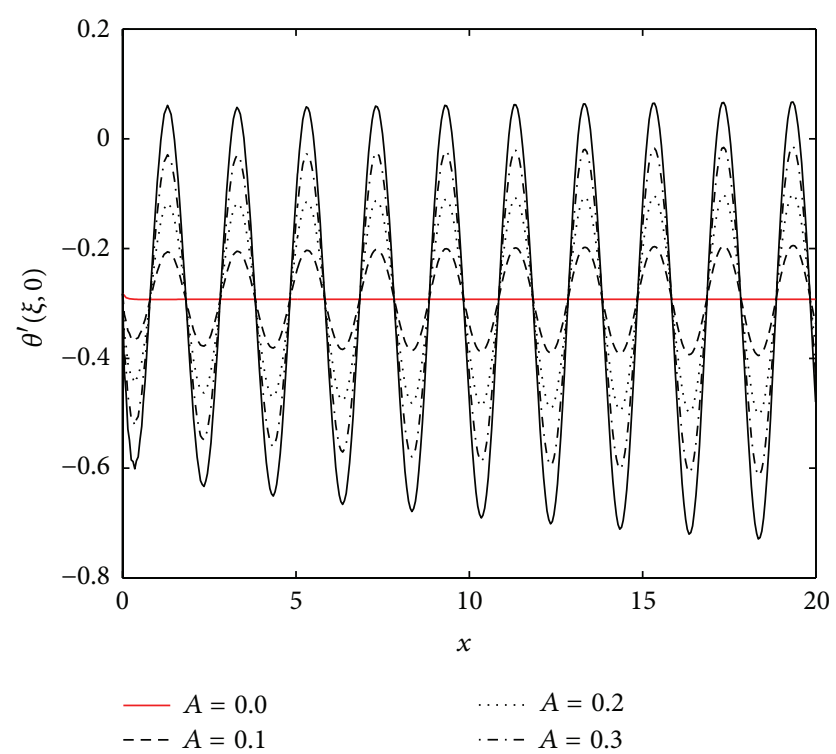

(b)

Figure 7: Effect of the $A$ on $f^{\prime \prime}(x, 0)$ and $\theta^{\prime}(x, 0)$ for $\mathrm{Le}=10, \mathrm{Pr}=1, \sigma=0.02, \mathrm{Nr}=0.1, \mathrm{Nt}=0.3$, and $\mathrm{Nb}=0.5$.

TABLE 1: Comparisons of wall skin-friction coefficient $f^{\prime \prime}(0)$ and local Nusselt number $-\theta^{\prime}(0)$ with those of Rees [26] for various values of Prandtl number Pr with $\mathrm{Nt}=0, \mathrm{Nb}=0, \mathrm{Nr}=0$, and $\sigma=0$.

\begin{tabular}{lcccc}
\hline Pr & \multicolumn{2}{c}{ Rees [26] } & \multicolumn{2}{c}{ Present results } \\
\hline 0.50 & $f^{\prime \prime}(0)$ & $-\theta^{\prime}(0)$ & $f^{\prime \prime}(0)$ & 0.31195217 \\
0.70 & 1.0086 & 0.3120 & 1.00855006 & 0.35320776 \\
1.00 & 0.9601 & 0.3532 & 0.96012412 & 0.40103314 \\
1.50 & 0.9082 & 0.4010 & 0.90819121 & 0.46070468 \\
2.00 & 0.8492 & 0.4607 & 0.84923678 & 0.50661849 \\
3.00 & 0.8079 & 0.5066 & 0.80788807 & 0.57667739 \\
4.00 & 0.7508 & 0.5767 & 0.75076470 & 0.63042597 \\
5.00 & 0.7113 & 0.6304 & 0.71128220 & 0.67458252 \\
6.00 & 0.6814 & 0.6746 & 0.68135380 & 0.71234690 \\
7.00 & 0.6574 & 0.7123 & 0.65739280 & 0.74551232 \\
8.00 & 0.6375 & 0.7455 & 0.63749977 & 0.77519223 \\
10.00 & 0.6206 & 0.7752 & 0.62054992 & 0.82684396 \\
20.00 & 0.5928 & 0.8268 & 0.59283303 & 1.00519108 \\
40.00 & 0.5119 & 1.0052 & 0.51189002 & 1.21460776 \\
70.00 & 0.4392 & 1.2146 & 0.43918933 & 1.41042858 \\
100.00 & 0.3866 & 1.4104 & 0.38663181 & 1.54953322 \\
\hline
\end{tabular}

the velocity decreases with the increase of $\sigma$. The influence of buoyancy parameter $\mathrm{Nr}$ on the local temperature and nanoparticle profiles is displayed in Figure 2. It can be noticed that both the thermal and the nanoparticle boundary layers thicknesses increase with an increase in $\mathrm{Nr}$, so the local temperature and nanoparticle volume fraction profiles are seen to increase when $\mathrm{Nr}$ increases. Figure 3 depicts the influence of the nonlinear density temperature variation on the temperature and the nanoparticle volume fraction profiles in the boundary layer. From the graphical representation it is apparent that the fluid is brought closer to the surface and reduces the thermal and nanoparticle volume boundarylayer thickness with increasing $\sigma$. Hence, when $\sigma$ increases there will be a decrease in the temperature profile and in the nanoparticle volume fraction.

Figure 4 illustrates the effects of the thermophoresis and Brownian motion parameters on the fluid velocity $f^{\prime}(\eta)$. We observe that the velocity of the nanofluid decreases as the 


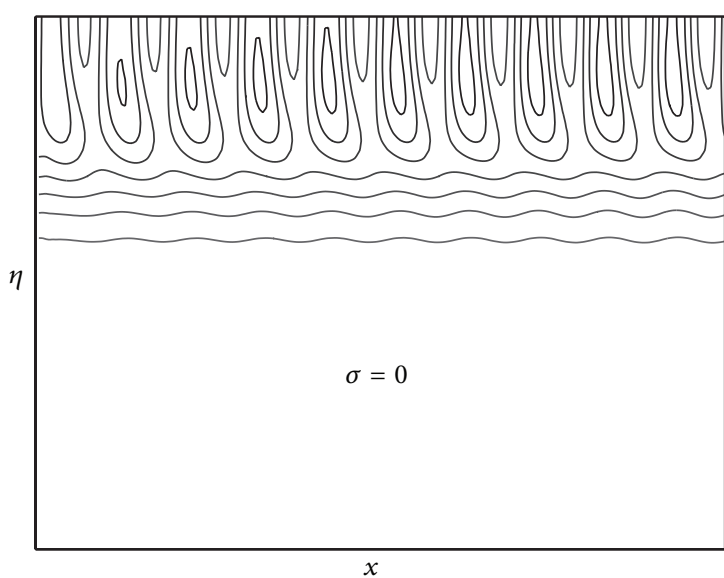

(a)

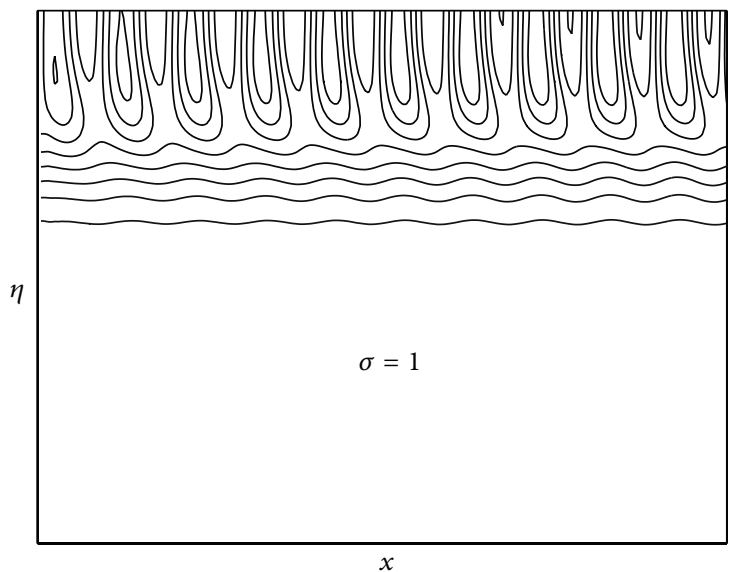

(c)

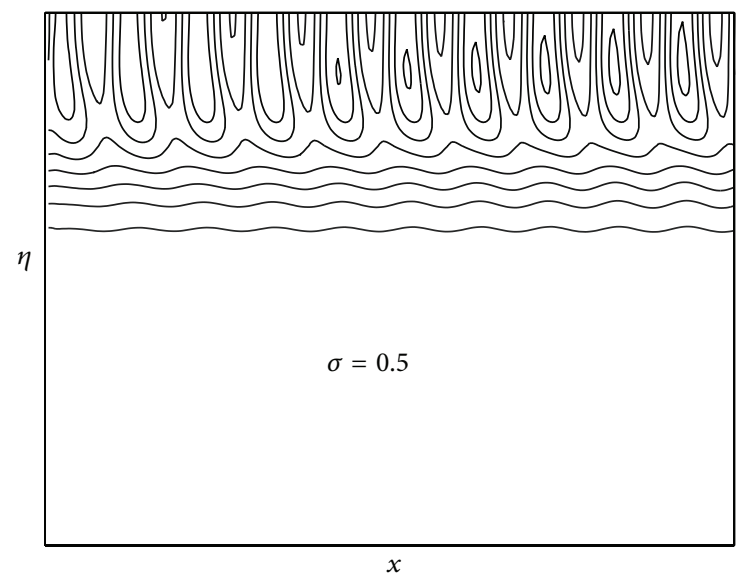

(b)

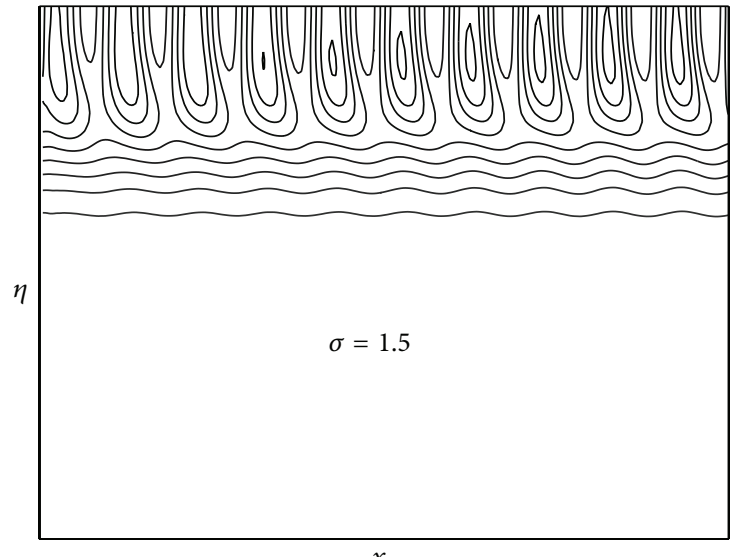

(d)

Figure 8: $\mathrm{Le}=1, \operatorname{Pr}=1, A=0.1, \mathrm{Nr}=0.1, \mathrm{Nt}=0.1$, and $\mathrm{Nb}=0.5$.

thermophoresis parameter Nt increases; however, the Brownian motion parameter $\mathrm{Nb}$ makes the velocity component $f^{\prime}(\eta)$ increase. As expected, the boundary-layer thickness of the momentum decreases with increasing $\mathrm{Nt}$, whereas the opposite behaviour of the velocity component $f^{\prime}(\eta)$ is observed with increasing $\mathrm{Nb}$. The effect of thermophoresis parameter on the temperature and mass volume fraction profiles is shown in Figure 5. The thermophoresis force generated by the temperature gradient creates a fast flow away from the surface; hence more fluid is heated away from the surface, and, consequently, as Nt increases, the temperature within the boundary layer increases. The flow from the surface carries with it nanoparticles leading to increase in the mass volume fraction boundary-layer thickness. The effect of Brownian motion on the temperature and nanoparticle volume fraction has been plotted in Figure 6. The Brownian motion represents the random movement caused by the nanoparticles that are suspended within the fluid. The local temperature increases as the Brownian motion parameter $\mathrm{Nb}$ increases; hence the Brownian motion is an important mechanism for the enhancement of thermal conductivity of nanofluids. However, Brownian motion leads to repositioning of the nanoparticles away from the fluid regime which causes a clustering near the surface. This leads to a decrease in the mass volume fraction.

Figure 7 shows effects of the temperature wave amplitude $A$ on both the scaled surface shear stress and the rate of surface heat transfer, respectively. As the temperature wave amplitude increases, the thickness of the momentum boundary layer increases; thus we can expect a decrease in the scaled surface shear stress. However, the fast flow from the surface increase leads to thinness in the boundary layer and hence increases the heat transfer rate. Thus the local temperature increases with increasing $A$; the same result has been reported by Rees [26] for regular fluids.

It is clear from Figure 8 that, as the streamwise distance $x$ increases, there is an increase in the size of the inner isotherms cells while there is no variation in the outer cells. However increases in the nonlinear temperature parameter $\sigma$ create more cells. This is because the thickness of the boundary layer varies with increasing both $x$ and $\sigma$. Figure 9 shows the isotherms for several values of the temperature wave 


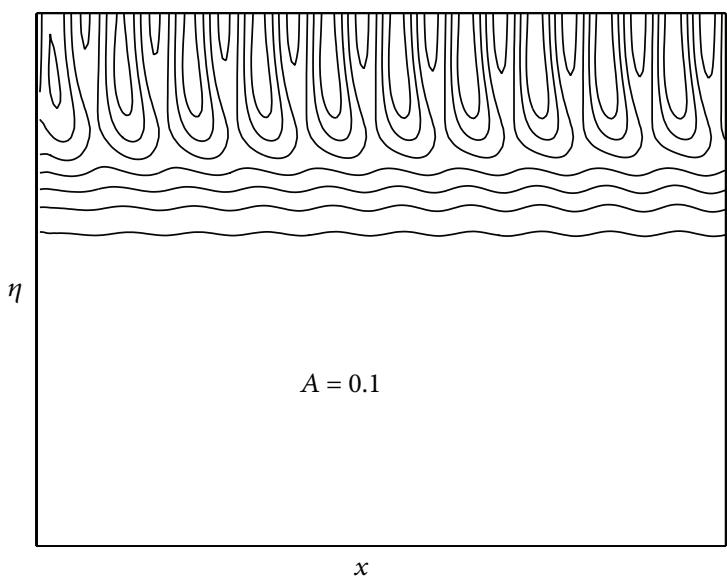

(a)

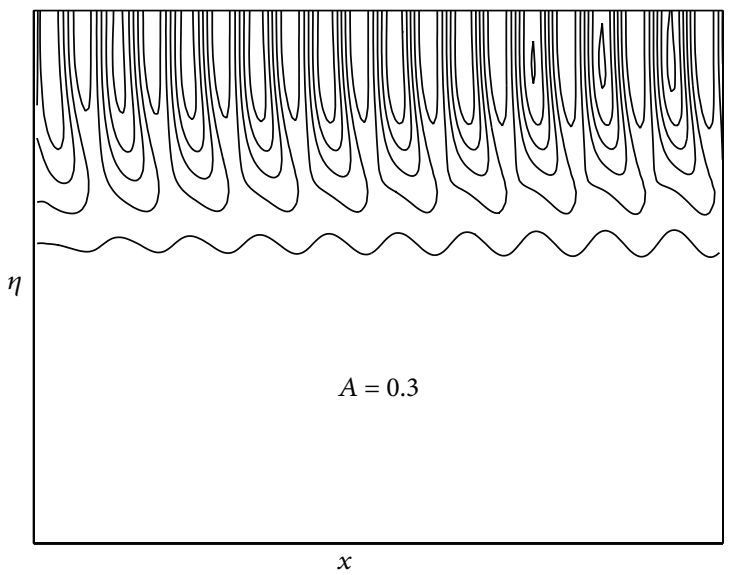

(c)

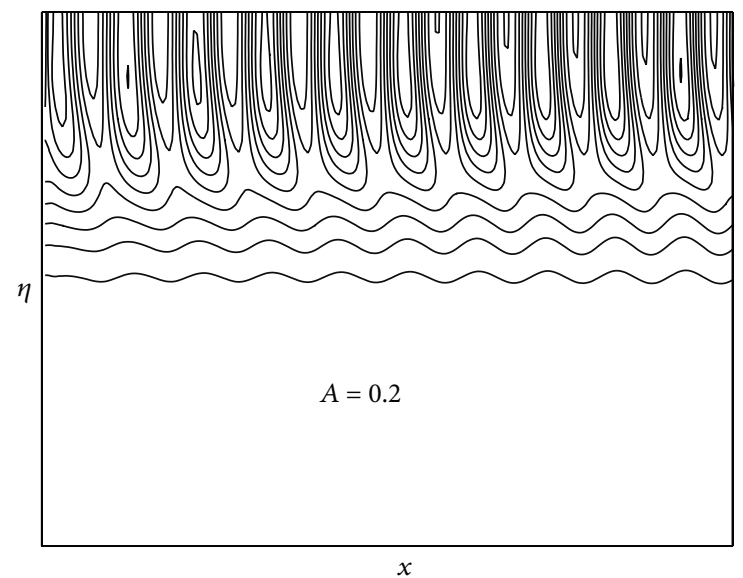

(b)

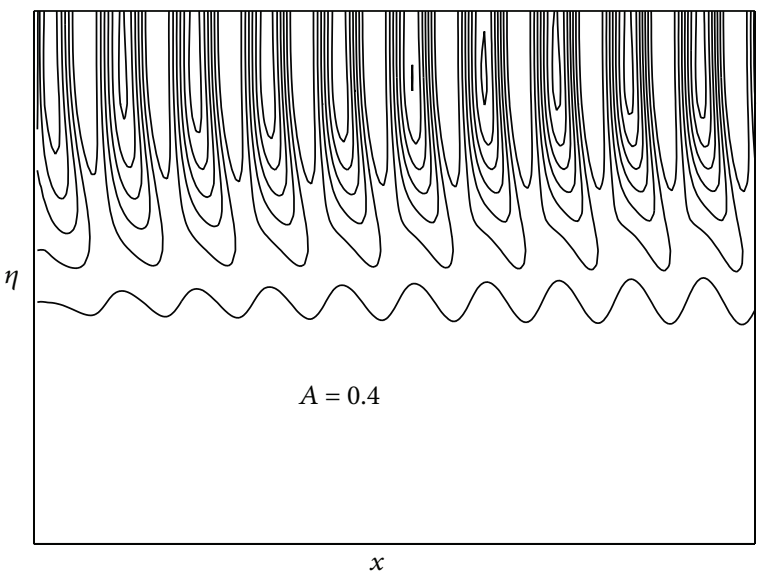

(d)

Figure 9: $\mathrm{Le}=1, \operatorname{Pr}=1, A=0.1, \mathrm{Nr}=0.1, \mathrm{Nt}=0.1$, and $\mathrm{Nb}=0.5$.

amplitude $A$. The variation of temperature wave amplitude leads to an increase in boundary-layer thickness, so that the inner isotherms cell size increases and more inner isotherms cells are created.

\section{Conclusions}

In this investigation, the problem of nonlinear nanofluid flow over heated vertical surface with sinusoidal wall temperature variations has been considered. The model includes the effects of Brownian motion and thermophoresis effects. Numerical approximations for the coupled high nonlinear partial differential equations governing were obtained using the local linearisation method. The effects of various parameters on the fluid properties and on the scaled surface shear stress and rate of surface heat transfer were determined. It was found that as the nonlinear temperature parameter increases the velocity tends to increase very closely to the surface and then decreases. Moreover, it was observed that the boundarylayer thickness of the momentum decreases with an increase in the thermophoresis parameter. The random movement caused by the nanoparticles enhanced the temperature within the boundary layer when increased.

\section{Conflict of Interests}

The authors declare that there is no conflict of interests regarding the publication of this paper.

\section{References}

[1] S. U. S. Choi, "Enhancing thermal conductivity of fluids with nanoparticles," in Proceedings of the International Mechanical Engineering Congress and Exhibition, FED 231/MD, pp. 99-105, ASME, San Franciscos, Calif, USA, 1995.

[2] K. V. Wong and O. De Leon, "Applications of nanofluids: current and future," Advances in Mechanical Engineering, vol. 2010, Article ID 519659, 11 pages, 2010.

[3] J. A. Gbadeyan, M. A. Olanrewaju, and P. O. Olanrewaju, "Boundary layer flow of a nanofluid past a stretching sheet with a convective boundary condition in the presence of magnetic field and thermal radiation," Australian Journal of Basic and Applied Sciences, vol. 5, no. 9, pp. 1323-1334, 2011.

[4] R. Sharma, A. Ishak, and I. Pop, "Partial slip flow and heat transfer over a stretching sheet in a nanofluid," Mathematical Problems in Engineering, vol. 2013, Article ID 724547, 7 pages, 2013. 
[5] Y. Ding, H. Chen, L. Wang et al., "Heat transfer intensification using nanofluids," Kona, vol. 25, pp. 23-38, 2007.

[6] J. Buongiorno, "Convective transport in nanofluids," Journal of Heat Transfer, vol. 128, no. 3, pp. 240-250, 2006.

[7] A. V. Kuznetsov and D. A. Nield, "Natural convective boundarylayer flow of a nanofluid past a vertical plate," International Journal of Thermal Sciences, vol. 49, no. 2, pp. 243-247, 2010.

[8] D. A. Nield and A. V. Kuznetsov, "The Cheng-Minkowycz problem for natural convective boundary-layer flow in a porous medium saturated by a nanofluid," International Journal of Heat and Mass Transfer, vol. 52, no. 25-26, pp. 5792-5795, 2009.

[9] W. A. Khan and I. Pop, "Boundary-layer flow of a nanofluid past a stretching sheet," International Journal of Heat and Mass Transfer, vol. 53, no. 11-12, pp. 2477-2483, 2010.

[10] C. J. Ho, W. K. Liu, Y. S. Chang, and C. C. Lin, "Natural convection heat transfer of alumina-water nanofluid in vertical square enclosures: an experimental study," International Journal of Thermal Sciences, vol. 49, no. 8, pp. 1345-1353, 2010.

[11] W. A. Khan and A. Aziz, "Double-diffusive natural convective boundary layer flow in a porous medium saturated with a nanofluid over a vertical plate: Prescribed surface heat, solute and nanoparticle fluxes," International Journal of Thermal Sciences, vol. 50, no. 11, pp. 2154-2160, 2011.

[12] A. M. Rashad, M. A. El-Hakiem, and M. M. M. Abdou, "Natural convection boundary layer of a non-Newtonian fluid about a permeable vertical cone embedded in a porous medium saturated with a nanofluid," Computers \& Mathematics with Applications, vol. 62, no. 8, pp. 3140-3151, 2011.

[13] A. Aziz, W. A. Khan, and I. Pop, "Free convection boundary layer flow past a horizontal flat plate embedded in porous medium filled by nanofluid containing gyrotactic microorganisms," International Journal of Thermal Sciences, vol. 56, pp. 4857, 2012.

[14] P. Rana, R. Bè, and O. A. Bég, "Numerical solution for mixed convection boundary layer flow of a nanofluid along an inclined plate embedded in a porous medium," Computers and Mathematics with Applications, vol. 64, no. 9, pp. 2816-2832, 2012.

[15] M. Hajipour and A. Molaei Dehkordi, "Analysis of nanofluid heat transfer in parallel-plate vertical channels partially filled with porous medium," International Journal of Thermal Sciences, vol. 55, pp. 103-113, 2012.

[16] R. Chand and G. C. Rana, "On the onset of thermal convection in rotating nanofluid layer saturating a Darcy-Brinkman porous medium," International Journal of Heat and Mass Transfer, vol. 55, no. 21-22, pp. 5417-5424, 2012.

[17] V. N. Korovkin and A. P. Andrievskii, "Free convection with a nonlinear dependence of density on temperature: plane problems," Journal of Engineering Physics and Thermophysics, vol. 73, no. 2, pp. 381-386, 2000.

[18] K. Vajravelu, J. R. Cannon, J. Leto et al., "Nonlinear convection at a porous flat plane with application to heat transfer from a dike," Journal of Mathematical Analysis and Applications, vol. 277, no. 2, pp. 609-623, 2003.

[19] H. Barrow and T. L. S. Rao, "The effect of variable beta on free convection," British Chemical Engineering, vol. 16, pp. 704-709, 1971.

[20] V. L. Streeter, Handbook of Fluid Dynamics, vol. 6, McGraw-Hill, New York, NY, USA, 1961.

[21] K. Vajravelu and K. S. Sastri, "Fully developed laminar free convection flow between two parallel vertical walls-I," International Journal of Heat and Mass Transfer, vol. 20, no. 6, pp. 655-660, 1977.
[22] K. V. Prasad, K. Vajravelu, and R. A. Van Gorder, "Non-Darcian flow and heat transfer along a permeable vertical surface with nonlinear density temperature variation," Acta Mechanica, vol. 220, no. 1-4, pp. 139-154, 2011.

[23] S. S. Motsa, F. G. Awad, Z. G. Makukula, and P. Sibanda, "The spectral homotopy analysis method extended to systems of partial differential equations," Abstract and Applied Analysis, vol. 2014, Article ID 241594, 11 pages, 2014.

[24] S. Ostrach, "An analysis of laminar free-convection flow and heat transfer about a flat plate parallel to the direction of the generating body force," NACA, vol. 2635, 1952.

[25] E. M. Sparrow and J. L. Gregg, "Similar solution for free convection from a non-isothermal vertical plate," Journal of Heat Transfer, vol. 80, pp. 379-384, 1958.

[26] D. A. S. Rees, "The effect of steady streamwise surface temperature variations on vertical free convection," International Journal of Heat and Mass Transfer, vol. 42, no. 13, pp. 2455-2464, 1999.

[27] I. Pop and D. B. Ingham, Convective Heat Transfer: Mathematical and Computational Modelling of Viscous Fluids and Porous Media, Pergamon, Oxford, UK, 2001.

[28] N. C. Roy and M. A. Hossain, "Numerical solution of a steady natural convection flow from a vertical plate with the combined effects of streamwise temperature and species concentration variations," Heat and Mass Transfer, vol. 46, no. 5, pp. 509-522, 2010.

[29] S. C. Saha, J. C. Patterson, and C. Lei, "Natural convection and heat transfer in attics subject to periodic thermal forcing," International Journal of Thermal Sciences, vol. 49, no. 10, pp. 1899-1910, 2010.

[30] M. M. Molla, S. C. Saha, and M. A. Hossain, "Radiation effect on free convection laminar flow along a vertical flat plate with streamwise sinusoidal surface temperature," Mathematical and Computer Modelling, vol. 53, no. 5-6, pp. 1310-1319, 2011.

[31] P. Rana and R. Bhargava, "Flow and heat transfer analysis of a nanofluid along a vertical flat plate with non-uniform heating using fem: effect of nanoparticle diameter," International Journal of Applied Physics and Mathematics, vol. 1, pp. 171-176, 2011.

[32] S. S. Motsa, "A new spectral local linearization method for nonlinear boundary layer flow problems," Journal of Applied Mathematics, vol. 2013, Article ID 423628, 15 pages, 2013.

[33] S. S. Motsa, Z. G. Makukula, and S. Shateyi, "Spectral local linearisation approach for natural convection boundary layer flow," Mathematical Problems in Engineering, vol. 2013, Article ID 765013, 7 pages, 2013.

[34] C. Canuto, M. Y. Hussaini, A. Quarteroni, and T. A. Zang, Spectral Methods in Fluid Dynamics, Springer Series in Computational Physics, Springer, Berlin, Germany, 1988.

[35] L. N. Trefethen, Spectral Methods in MATLAB, SIAM, New York, NY, USA, 2000. 


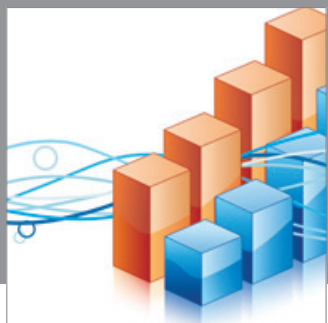

Advances in

Operations Research

mansans

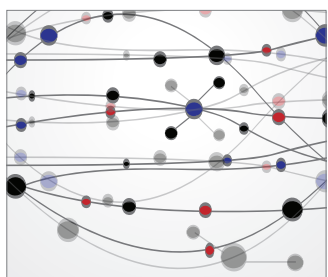

The Scientific World Journal
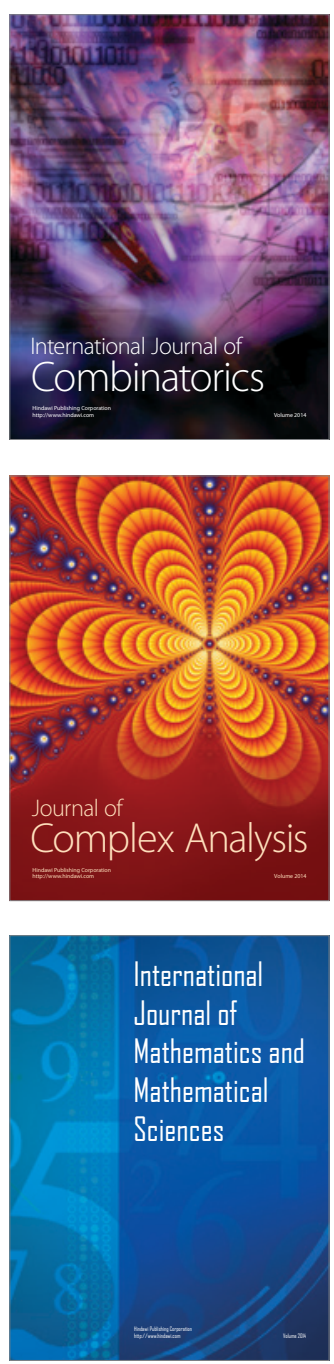
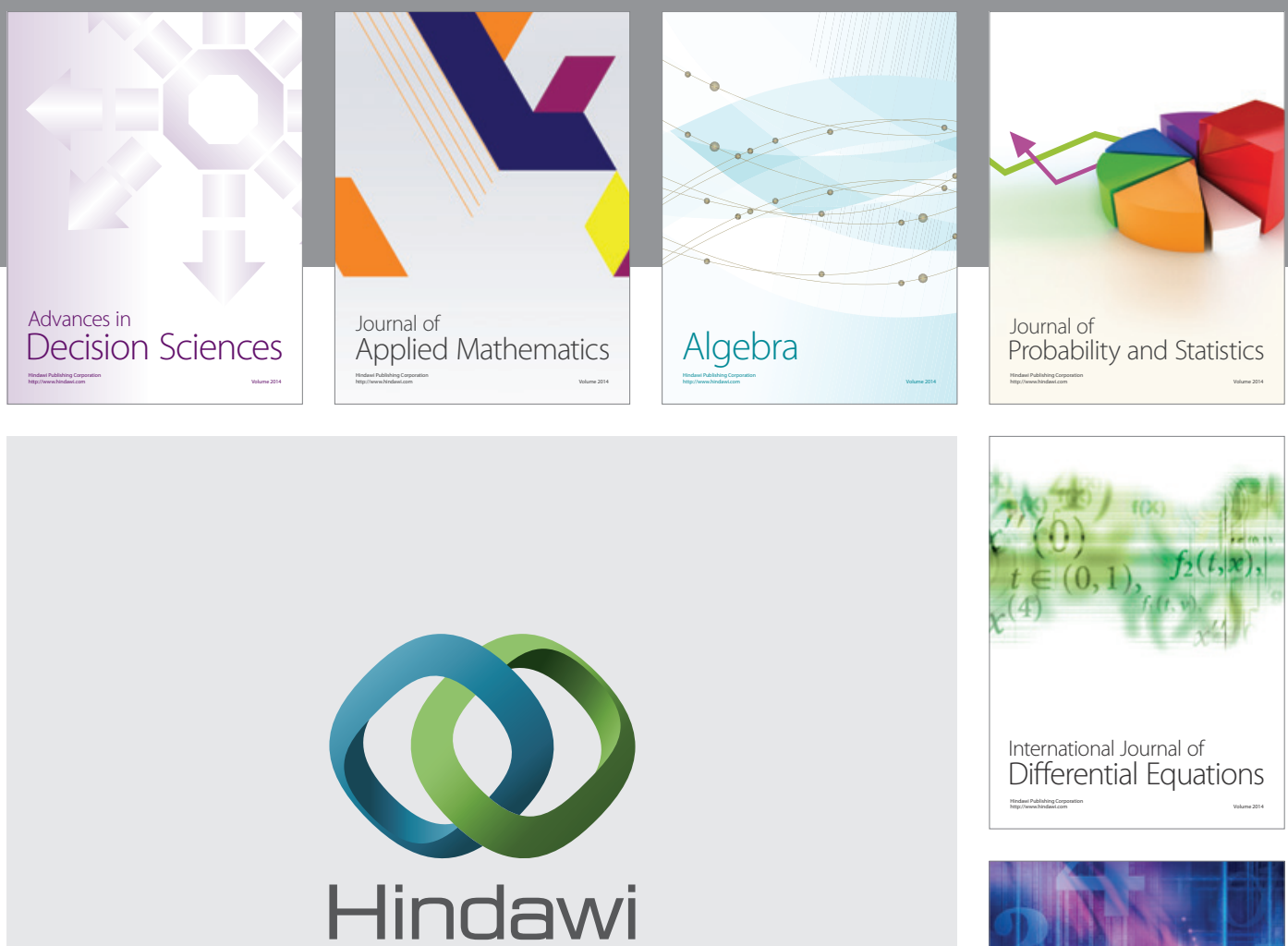

Submit your manuscripts at http://www.hindawi.com
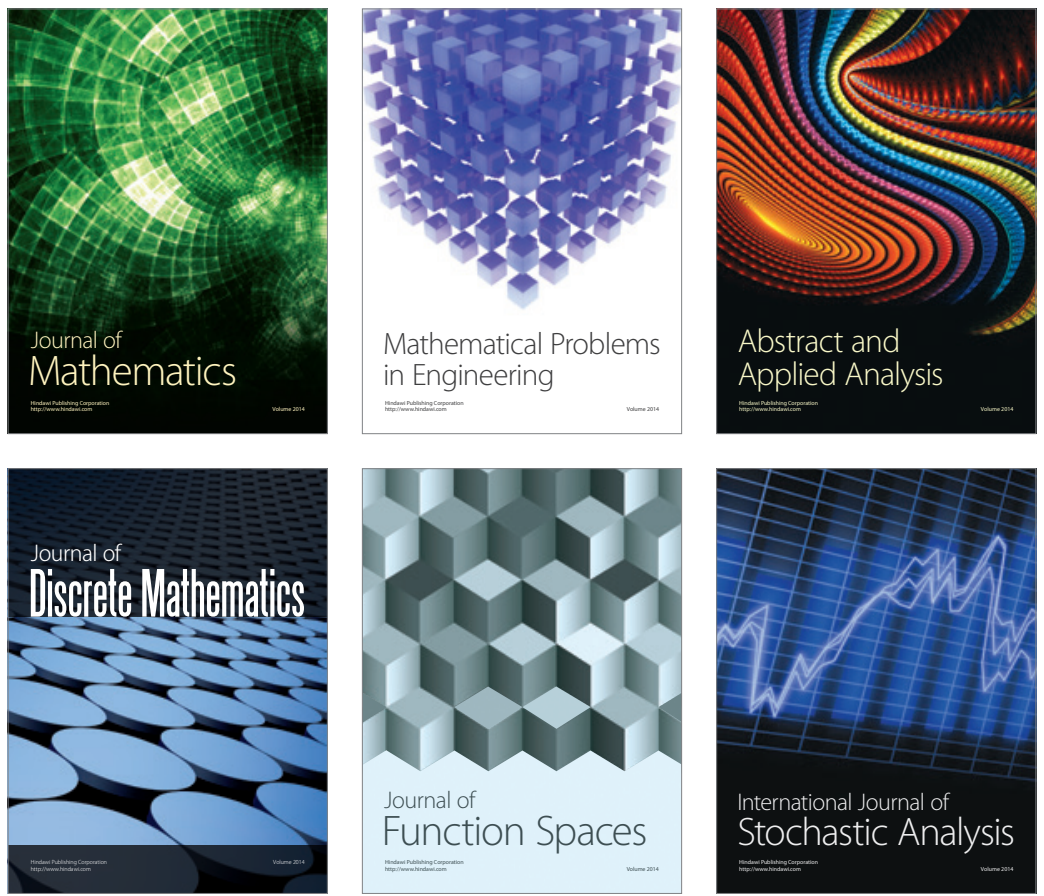

Journal of

Function Spaces

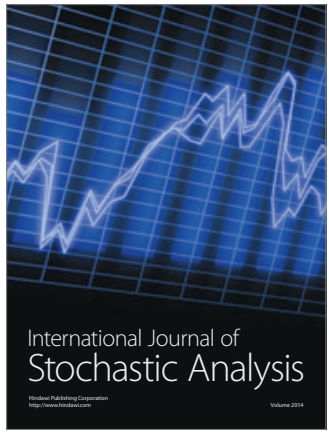

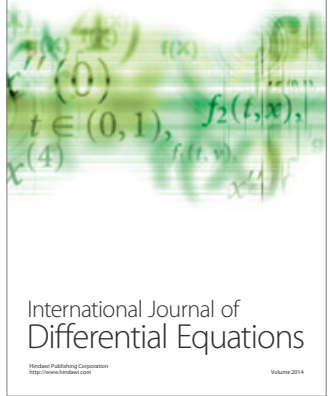
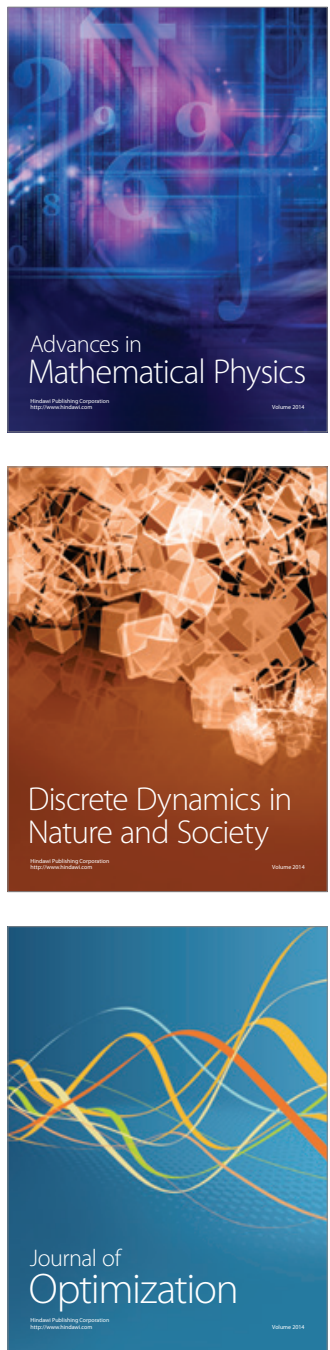